\title{
Research and Design of Single - Phase Boost PFC Circuit Based on Current Hysteresis
}

\author{
Yongjie Yu \\ North China Electric Power University, Beijing, 102206 \\ 18811337377@163.com
}

Keywords: Active Power Factor Correction; Current Hysteresis; PI Regulator; THD Loss Analysis

\begin{abstract}
Traditional rectifier circuit by the diode or thyristor, work to produce a large number of harmonics. Active power factor correction (PFC) on this basis to add high-performance full-control power electronic devices, such as MOSFET and IGBT, and control the input current to make it close to the sine wave and the unit power factor. In recent years, with the requirements of the performance of electrical equipment continues to improve, this technology has become a hot spot in the field of power electronics research, and has been widely practical application.

For the single-switch and DC-side Boost-type boost circuit, this paper uses the current hysteresis control strategy and PI regulator on the DC side of the fixed voltage control. Analyze the continue current mode (CCM) and the discontinue current mode (DCM). Then the harmonic analysis and switching loss analysis are carried out for the two models.

Through the PSCAD simulation, total harmonic distortion (THD) of the current harmonics is low by the discrete Fourier transform (FFT) of the AC current waveforms of the two models. In this paper, we use parallel switch on the buffer circuit to simulate the switching losses, DCM control can be a good way to reduce the opening loss of the switch.
\end{abstract}

\section{Introduction}

Single-phase full-bridge controllable rectifier circuit consists of four half-controlled thyristor, by changing the trigger angle to adjust the rectifier side voltage. The disadvantage is the consumption of a large number of reactive power and produce harmonic interference, and the trigger angle adjustment range is limited, the rectifier side voltage range is too limited ${ }^{[1]}$.

In order to solve the above problems, we can use PWM rectifier circuit, that is, all four thyristors replaced by full-control IGBT devices, but compared to thyristors, IGBT expensive and relatively small capacity, the higher the cost of circuit transformation. With the need to rectify the DC drive to drive a substantial increase in power equipment as well as the international restrictions on the implementation of power harmonics standards. Active power factor correction technology has become a hotspot in the field of power electronics, which can add a small amount of full control device to the sine wave current and the unit power factor on the basis of the original uncontrollable or semi-controlled rectifier circuit.

As shown in Fig 1, when the controllable switch V is on, the power supply $\mathrm{E}$ is charged to the inductor $\mathrm{L}$ while the capacitor $\mathrm{C}$ supplies power to the load. As the value of $\mathrm{C}$ is large, the output voltage $\mathrm{U} 0$ is kept constant. When $\mathrm{V}$ is in the off state, $\mathrm{E}$ and $\mathrm{L}$ collectively charge the capacitor and supply energy to the load R. Boost type has been able to make the output voltage higher than the power supply voltage, the key for two reasons: First, the inductance L after the energy storage has the role of voltage pump, and second, the capacitor $\mathrm{C}$ can maintain the output voltage.

As shown in Fig 2, in the rectifier circuit DC side by adding Boost module, you can meet the following conditions ${ }^{[2]}$ :

1) Boost can meet the voltage transmission ratio greater than 1 , that is, with the ability to boost.

2) The input current is controllable and tracks the AC voltage waveform.

3) The amplitude of the AC side is properly adjusted so that the output voltage is stabilized at the required voltage. 


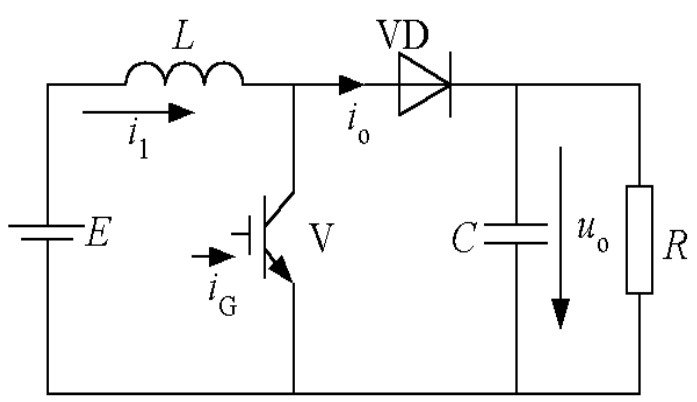

Figure 1 Boost Circuit

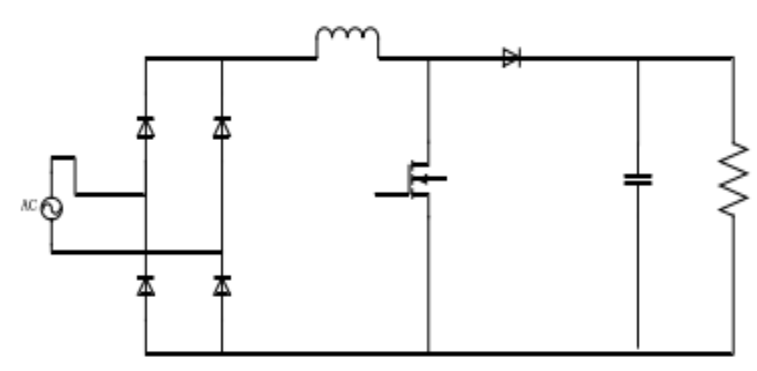

Figure 2 Boost PFC

\section{Modeling of Single - Phase Boost PFC Circuit}

The working status of CCM is shown in Figure 3.In the continuous mode, the instantaneous value of the AC input current is used to track the instantaneous value of the AC voltage of the grid and the current ripple is also small. This not only reduces the design volume of the filter, but also greatly reduces the current peak of the switch tube ${ }^{[3][4]}$.

The working status of DCM is shown in Figure 4. It is characterized by the inductor energy can be fully transmitted, in each switching cycle, the boost inductor from the input power to draw the energy completely transferred to the output capacitor into the storage capacity. At this time the current itself can track the phase angle of the sinusoidal voltage, get a higher power factor

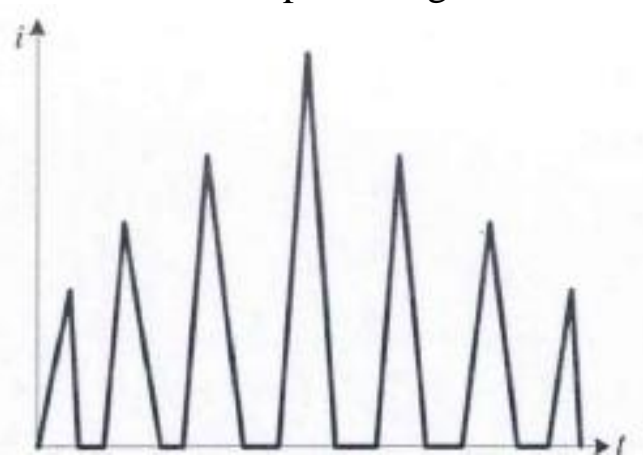

Figure 3 CCM Mode

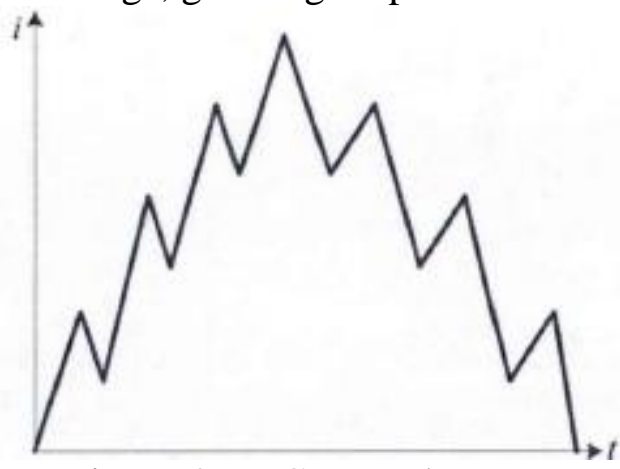

Figure 4 DCM Mode

\section{Modeling of CCM}

Assuming that the current in the inductor is continuous for a period, that is, the inductor current does not exist for zero interval, the parallel capacitor is large enough, the DC side voltage is approximately constant, ignoring the influence of the resistor.

\section{Switching Cycle Analysis}

Phase 1: Inductor storage magnetic energy stage

IGBT in the conduction state, the diode is off, because the voltage and current in phase can be equivalent to the $\mathrm{AC}$ side of the $\mathrm{AC}$ side of the $\mathrm{DC}$ side, set the $\mathrm{AC}$ side voltage, the $\mathrm{DC}$ side voltage $^{[5]}$. The voltage on the inductor is

$$
V_{L}=V_{d}(w t)=\sqrt{2} V_{s}|\sin w t|
$$

According to Faraday's law of electromagnetic induction, the inductance current change rate is obtained

$$
\frac{d i_{L}}{d t}=\frac{\sqrt{2} V_{s}}{L}|\sin w t| \geq 0
$$

The above equation shows that in phase 1 , the inductor current is in the rising state, and the rise rate is sinusoidal Variety.

Phase 2: Inductance release magnetic energy phase IGBT is in the off state, and the diode is in the open state, the inductor is in the release of magnetic 
energy stage. Set the voltage at both ends of the capacitor. The voltage is applied to the input inductance $\mathrm{L}$, the inductor voltage is

$$
V_{L}=V_{d}(w t)-V_{0}=\sqrt{2} V_{0}|\sin w t|-V_{0}
$$

The current change rate of inductance $L$ is

$$
\frac{d i_{L}}{d t}=\frac{\sqrt{2} V_{s}|\sin w t|-V_{0}}{L}<0
$$

The above equation shows that in phase 2, the inductor current drops, and the inductance L, along with the input supply, supplies power to the output load and charges the capacitor C. However, due to the continuous current, the magnetic energy stored in the inductor $L$ is not exhausted in phase 2 , that is, the value of the inductor current at phase 2 is greater than zero.

The above analysis shows that the rate of change of the inductor current varies with the phase angle. When 0 degrees, the input grid voltage transient zero, phase 1 inductor current rise rate is equal to zero; and phase 2 of the rate of decline is high. When 90 degrees, the input voltage reaches the peak, stage 1 inductor current rise rate is high, but the rate of decline is slightly lower.

\section{Modeling of DCM}

Relative to the current continuous type, the current intermittent type when the switch is turned on when the inductor current rises from zero to the given value, the switch is turned off until the inductor current drops to zero to start the next switching cycle, so the inductor current waveform by a series Triangular wave composition,.

Assuming the triangular wave current frequency is very high, in each cycle that the AC voltage is basically the same, take the cycle of the corresponding time corresponding to the voltage, set the current AC current reference. According to the calculation of each cycle of the opening, turn off time.

$$
\begin{aligned}
& T_{\text {on }}=\frac{I_{s} L}{V_{s}} \\
& T_{\text {off }}=\frac{1}{\frac{V_{0}}{\sqrt{2} V_{s}|\sin w t 0|}-1} T_{o n}
\end{aligned}
$$

The above equation shows that the opening time of each cycle is the same, the turn-off time is different, with the corresponding value of the voltage corresponding to the cycle changes, the corresponding voltage absolute value, the longer the turn-off time, the longer the cycle time.

\section{Control Strategy}

\section{Current Hysteresis}

This paper uses the current hysteresis control mode ${ }^{[6][7]}$. The output current is used as the command signal, the actual current as a feedback signal, through the two instantaneous value comparison to determine the switching device on and off, so that the actual output tracking command signal changes.

As can be seen from the figure, when the AC side current reaches the upper limit, the IGBT turns off, the diode turns on and the current drops; when the current reaches the lower limit, the IGBT turns on and the current rises. By controlling the size of the inductor L to control the current rate of change, because the current rate of change is much larger than the frequency of sinusoidal current changes, the value of the inductance $L$ to be smaller. The lower the difference, the lower the switching frequency, because the hysteresis control is variable frequency control, through the difference control switching frequency is only a rough range.

\section{PI Regulator}

In order to ensure that the output DC voltage is constant and given the value. Since the value of the parallel capacitor $\mathrm{C}$ is large, the voltage can be kept constant. Where the equivalent resistance is 
used to load, according to the law of conservation of energy, you can adjust the AC side of the current amplitude to change the input power, without considering the switching losses, power consumption in the load. So if the actual voltage is less than the specified voltage, you can increase the current to boost, and vice versa can reduce the current to decompression. PI adjustment in the proportion of the adjustment by the size of the voltage difference to adjust the current, then add the integral adjustment can be adjusted to make the output voltage to the specified value.

\section{Simulation and Analysis}

Fig 5,6 for the simulation results, we can see that it is divided into two stages, the stage of a large current charge for the capacitor to the specified value of $200 \mathrm{~V}$; stage two for the constant unit power factor for the continuous power supply. It can be seen that the DC side voltage is not larger than the AC side during the high current charging process, so that the inductor current rises and the hysteresis loop does not work. Since the PI adjustment has overshoot, the value of the command current is almost reduced to zero when the voltage is overshoot and greater than the specified voltage. Therefore, there is a current region with a magnitude close to zero in the transient to steady state. After reaching the steady state, the hysteresis current control and the PI regulation work together so that the output voltage is maintained at a specified value of $200 \mathrm{~V}$ and hardly fluctuates.

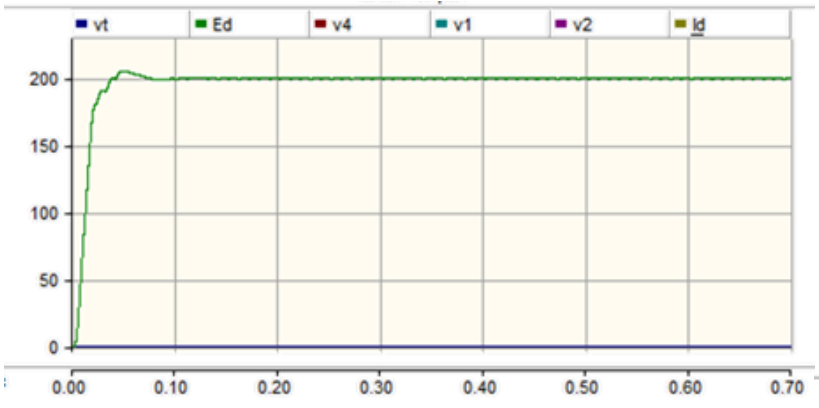

Figure 5 AC Input Current

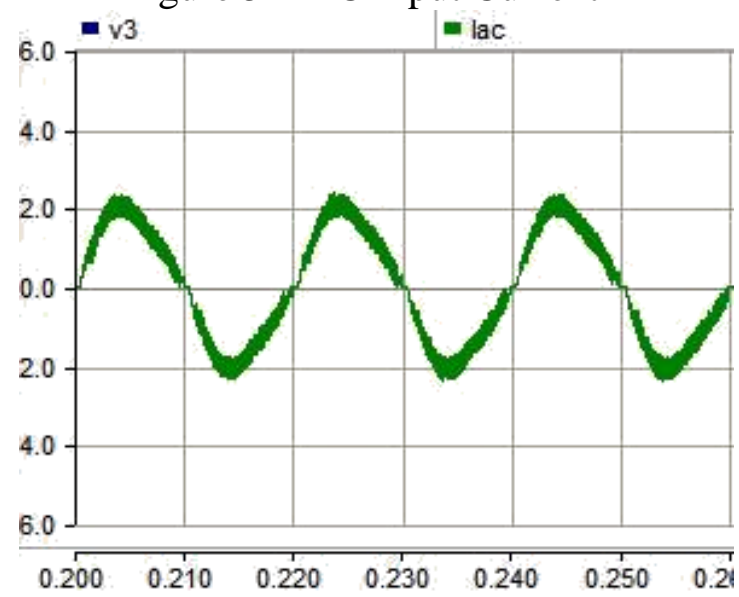

Figure 7 Current of CCM

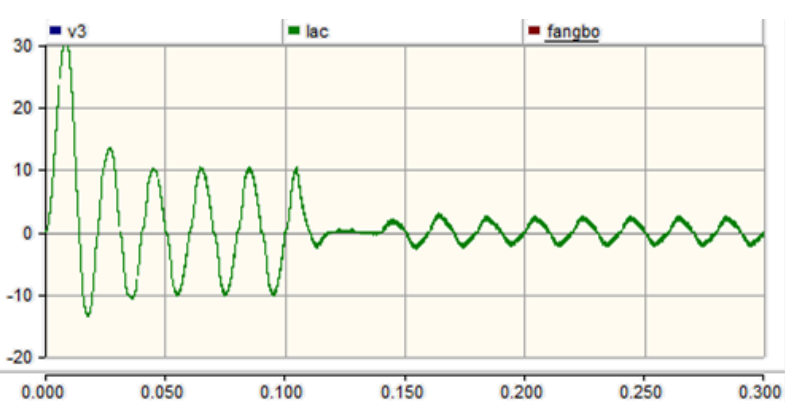

Figure 6 DC side Output Voltage

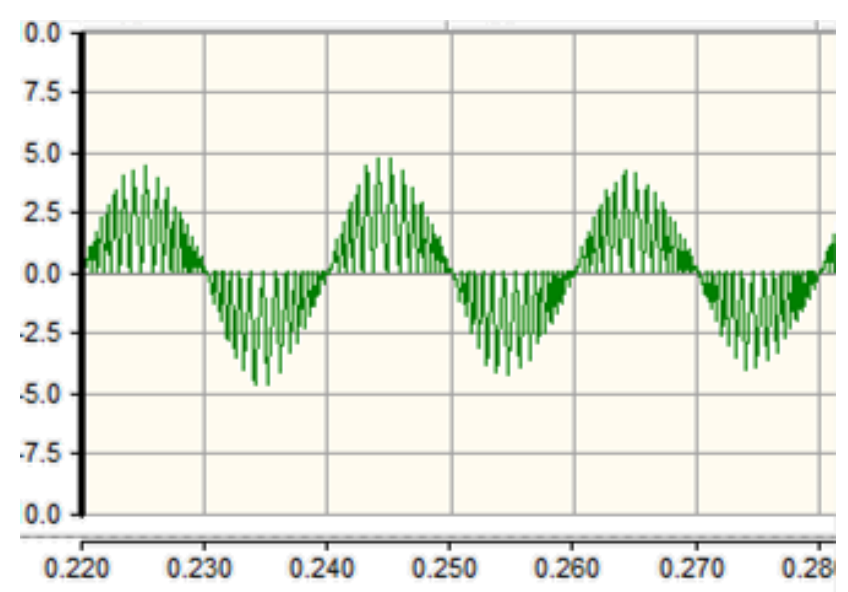

Figure 8 Current of DCM

\section{Harmonic}

The above figures show the AC current generated by two different control methods, which all satisfy the unit power factor, and the following is the harmonic analysis. ${ }^{[8][9]}$

Table 1 Analysis of Harmonic

\begin{tabular}{|c|c|c|c|c|c|c|c|}
\hline Harmonic & 1 & 2 & 3 & 4 & 5 & 6 & 7 \\
\hline CCM & 1.382 & 0.001 & 0.145 & 0.003 & 0.013 & 0.003 & 0.014 \\
\hline DCM & 1.387 & 0.014 & 0.075 & 0.017 & 0.015 & 0.010 & 0.017 \\
\hline
\end{tabular}


Compared to the fundamental frequency components, due to the same load, the fundamental frequency RMS is roughly equal, but the intermittent base frequency current maximum amplitude of the total current half of the maximum amplitude. As for the high harmonics are relatively small, can be ignored, the reason is the switching frequency is too high and are variable frequency control, there is no high harmonic situation.

\section{Loss Analysis}

Here we first analyze the switching losses, as shown in Fig 9, IGBT in the opening or closing process, both ends of the voltage and flow through its non-zero overlap time. ${ }^{[10]}$ After adding the snubber circuit, the IGBT is no longer an ideal switching device. When turned on, the IGBT voltage does not drop to zero, but undergo a gradual descent process. At the same time, the current through does not suddenly rise to the load current, there is also a rising time. During this time, there is an overlap between the voltage and current of the IGBT, and the resulting loss is called the turn-on loss. Similarly, when the IGBT is turned off, the voltage goes through a process from zero to the supply voltage, and the current flowing is gradually decreasing to zero. The loss caused by the overlapping area of this period is called turn-off loss. The sum of the opening loss and the turn-off loss is called the switching loss.

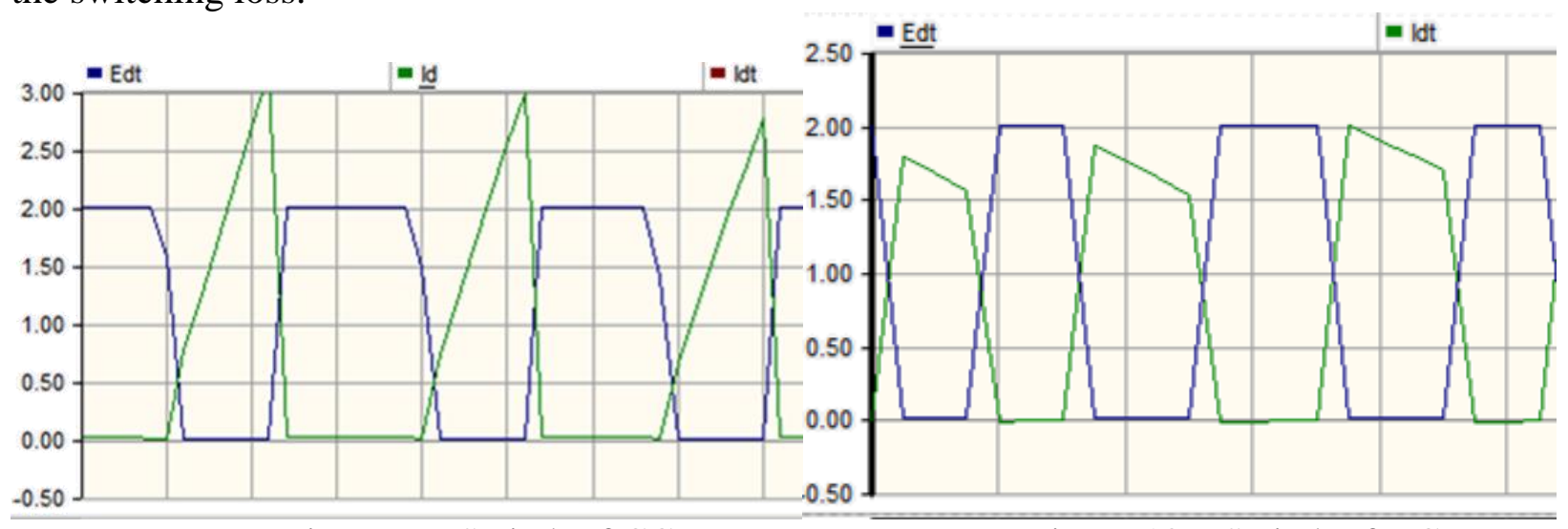

Figure 9 Switch of CCM

Figure $10 \quad$ Switch of DCM

Power factor correction technology switching frequency is higher, as the fundamental frequency of several times, so the switch loss is large, how small switching loss has become a research hotspot.

In order to reduce the switching losses, we use soft-switching technology, which by adding the resonant circuit to slow down the process of switching voltage and current changes, so that the switch before the opening voltage drop to zero, zero voltage open; Zero, zero current off.

Compared with the continuous and intermittent switch, it can be found that the voltage and current overlap area does not change and the switching loss is the same under the premise of not realizing the soft circuit of the resonant circuit. However, during the opening process, the current of the intermittent circuit is reduced to zero before it is turned on. When the turn-on voltage drops to zero, the current rises little and the current rises rapidly after the voltage drop is zero. The switching loss of the opening process is much smaller than that of the continuous circuit.

\section{Conclusion}

(1)In this paper, we use current hysteresis on the single-phase Boost PFC circuit control and the $\mathrm{AC}$ input current tracking voltage changes in the higher power factor, and the voltage overshoot is small, $0.1 \mathrm{~s}$ after entering a constant voltage, the system stability is good.

(2) The total current distortion at CCM and DCM is $11.1 \%$ and $15.2 \%$, showing that the current harmonics in CCM are less, and the peak current in DCM is almost twice that of CCM. The current peak will affect the equipment loss and service life.

(3) In DCM, IGBT switch open with zero current, greatly reducing the opening loss. Switching losses become $1 / 2$ of the original. 


\section{References}

[1] Wang Zhaoan, Liu Jinjun. Power Electronics Technology. Beijing: Mechanical Industry Press, 2008.

[2] Xu Dehong, etc .. Modern rectifier technology - active power factor correction technology, 2013.

[3] Garcia,Oscar,et al. Single phase power factor correction[J].IEEE Transactions on Power Electionics,2003(18.3): 749-755

[4] Wan Hui, Zhang Changdong, Huang Dan. Active power factor correction control technology status and development [J]. Electrical and electrical, 2013 (3): 1-6

[5] Su Juan, Cheng Jiebin, Jie Qian grass Based on the Boost converter topology PFC circuit modeling and analysis of the town of modern electronic technology, 2005 (15): 71-73

[6] LIANG Zhi-rui, YE Hui-qiang, ZHAO Fei. Overview on power system harmonic state estimation[J]. Power System Protection and Control, 2010(15): 157-159

[7] Wu Songrong, Zhang Fei, Xu Jianping, et al. Boost PFC converter with a new sinusoidal reference current algorithm[J]. IEEE, 2009(9): 711-715

[8] The algorithm of interpolating windowed FFT for harmonic analysis of electric power system. Fusheng Zhang,Zhongxing Geng,Wei Yuan. IEEE Transactions on Power Delivery . 2001

[9] Applications of the windowed FFT to electric power quality assessment. Heydt G T,Fjeld P S,Liu C C,et al. IEEE Transactions on Power Delivery . 1999

[10] IGBT device application aspects for $50-\mathrm{kW}$ zero-current-transition inverters. Li, Yong P.,Lee, Fred C.,Boroyevich, Dushan. IEEE Transactions on Industry Applications . 2004 